\title{
Design of Remote Monitoring System based on Embedded Web Server
}

\author{
Xiaohu Liu \\ Sunshine Boulevard No. 2666 college of Xinyu Xinyu, jiangxi, China \\ xylxh163@163.com
}

Keywords: Embedded; Web server; PLC; system; Remote monitoring

\begin{abstract}
The rapid development of information technology and the wide application of embedded systems have brought revolutionary changes to people's life. Based on the current production process and machine fault manufacturing process of the remote monitoring and control requirements, this article designed the remote monitoring system based on B/S system architecture, the embedded ARM platform and remote control side to create virtual dedicated channel, solve the problem of the network access equipment, the server USES HTTP protocol and CGI technology to complete the data processing and storage functions and realize the access, monitoring and maintenance of remote PLC equipment. This kind of monitoring system has good real-time performance, accurate data collection and friendly interface. It does not need to install specific software, and meets the needs of monitoring tasks. It has good cost performance and high use value.
\end{abstract}

\section{Introduction}

With the arrival of the network age, the network sharing of all kinds of information is more and more valued and favored by people. Connecting the industrial monitoring system to the Internet and realizing the remote monitoring and control of industrial field information has become a hot topic studied by domestic and foreign scholars. In this paper, an embedded remote monitoring system in $\mathrm{B} / \mathrm{S}$ mode is designed and implemented, taking the data collected by real-time monitoring industrial field PLC as an example. The hardware of this system adopts embedded controller and supports the Windows 2000 /XP operating system. Web server and database are installed on the server side to realize the storage of historical data. The client adopts DHTML technology to realize the human-computer interaction interface [1,2]. Using OPC technology, data communication between Web server and field PLC is realized. Using the system, as long as connected to the Internet, the user can through the browser anywhere monitoring information data of industrial field and control the state of field devices, and do not need to install any client software, greatly simplifies the client computer load, reduce the workload of system maintenance and upgrade costs, and, reduce the overall cost of users.

\section{Design of Remote Monitoring System}

The design of production site automation control system generally adopts the design principle of integration. The automatic control system for production control consists of two parts. Among them, the most basic equipment used for direct control of hardware equipment includes PLC, detection instrument, field limit, etc. In these basic control equipment, PLC is used as the control station to acquire and collect the operating conditions and control parameters of production field equipment for the control of production sequence according to the operation process of equipment. Other is the upper industrial monitoring operation equipment which includes industrial computer, PLC programming software and running monitoring software, the PLC programming software is used to write PLC program and configuration of PLC hardware and upload or download it to the normal operation of the PLC control equipment, monitoring software, according to the operational status of the production workshop production line configuration, realize the equipment running condition monitoring and control of production parameters have the generation of production report, application command control and production management information exchange, etc. 
The PLC workstation and detection equipment between the ipc and the lower control equipment constitute the industrial LAN and realize information communication through industrial Ethernet. The network supports the standard TCP/IP Ethernet protocol with a transmission rate of $10 / 100 \mathrm{~m}$ bit/s full duplex and automatic switching. The information exchange between PLC was carried out through industrial Ethernet, while the PLC adopted field distributed I/O structure. Since the controlled equipment is relatively large production equipment, the points to be collected and controlled by PLC should be dispersed to the field operation platform by using profibus-dp bus, and I/O station should be set in the field operation platform. Taking into account the network topology characteristics of PLC equipment and equipment hardware structure, automatic control equipment and hardware are connected by Ethernet [3,4], this paper adopts embedded ARM platform equipped with Ethernet module and 4G GPRS module to monitor equipment information, and its topology structure is shown in figure 1.

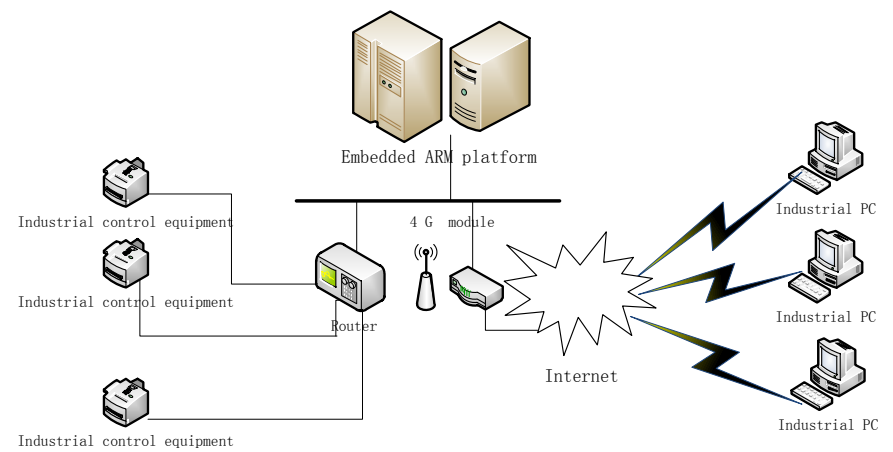

Figure 1. The overall design of the remote monitoring system

\section{Hardware design of remote monitoring system}

Embedded Web server remote monitoring system is mainly composed of client, embedded Web server and control field equipment. The PLC. The server is connected to the Internet through the router. The client only needs to enter the IP address of the server through the web browser on the computer that is already connected to the Internet to access the remote server [5,6]. The server communicates with PLC through Ethernet, so as to realize remote control of equipment. As an intermediate hub, embedded Web server is the key of system design. This system USES ARM S3C2440 as the controller of this system. The controller is suitable for all kinds of critical and harsh environments and meets the industrial requirements. CPU processing speed reaches $1.4 \mathrm{GHZ}$. Integrated $1 \mathrm{~GB}$ RAM, 512k B, battery backup RAM, able to save key data when the system power is off; There are two Ethernet ports of 10/100mbps, which can connect to the Internet using TCP/IP protocol, and also can realize Ethernet communication between the server and PLC. In addition, there are 2 rs-232 ports, 2 rs-232/422/485 ports and 4 USB ports for serial communication. Supports multiple operating systems. General diagram of system hardware structure is shown in figure 2.

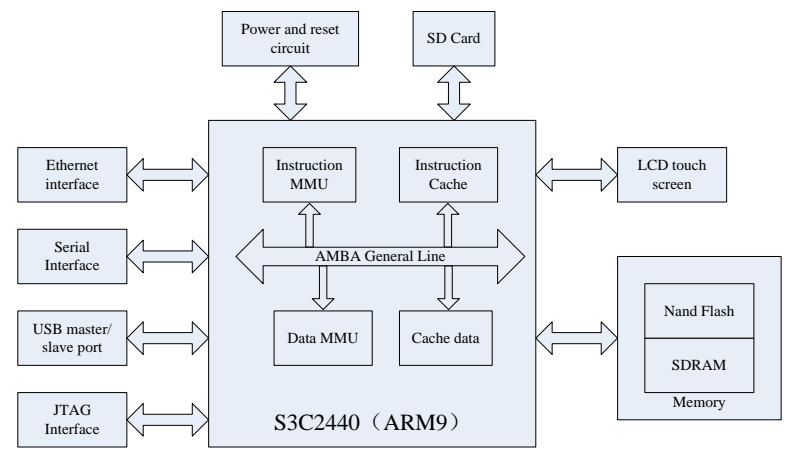

Figure 2. Diagram of system hardware structure

Embedded monitoring system software design

The system software design includes the virtual monitor network programming and the embedded Web server programming. 
Design of virtual special monitoring network program.Virtual private network VPN(virtual private network) is a private network technology that utilizes open public network to establish a dedicated data transmission channel. In essence, it is a network interconnection technology. The public network infrastructure meets enterprise interconnection needs and shares network resources, and it has the same guarantee of network users' security as private network. VPN can be built on the IP network of the Internet or Internet operators, as well as on the network infrastructure such as frame relay (FR) or asynchronous transmission and transmission mode (ATM), and the internal data of users can be transmitted safely on the public network through corresponding encryption and authentication technologies, so as to realize the exclusivity of network data transmission[7]. The working process of virtual special monitoring network is shown in figure 3.

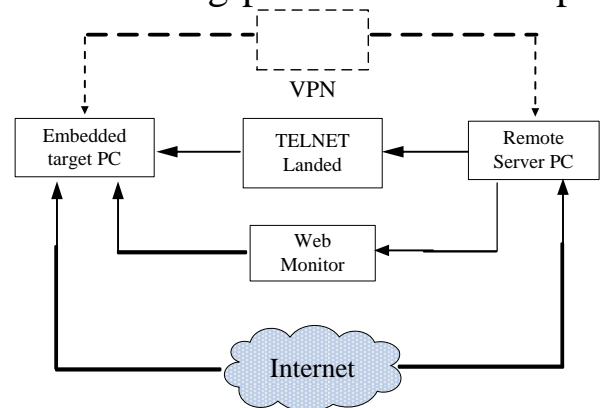

Figure 3. The working process of virtual special monitoring network

There are currently two different ways to implement VPNS: IPSec VPNS and Secure SocketsLayer(SSL) VPNS. According to the actual situation of the company, we must choose the best way to implement the company's security management. At present, most VPNS adopt IPSec's solution because IPSec provides encryption function for arbitrary data transmission, which can effectively protect IP packet security. With the rapid development of e-commerce and online banking, network security technology has made great progress, and SSL VPN is gradually replacing IPSec VPN in some applications. When users access the application server sends the request, the request has not been sent directly to the application server, but by the SSL VPN, after receiving the data by the SSL VPN protocol parsing in the first place, then execute the identity authentication and access control security strategy, eventually converts the data to the appropriate back-end agreement, transmitted to the application server. The dedicated network is effectively protected by allowing data flows into the application server after the security policy is executed.

OpenVPN provides a VPN solution that implements all the features of SSLVPN. Using OpenVPN, a tunnel connection can be established between any IP subnet or between any network card through a separate UDP or TCP port. And a scalable, load-balancing VPN server can be configured to handle thousands of dynamic connection requests from different VPN clients. OpenVPN provides encryption algorithms including DES, 3DES, BLOWFISH, and IDEA that use these encryption algorithms and authentication mechanisms to protect the transmission of data in VPNS over the Internet.

\section{Embedded Web server programming.}

Embedded Web server program is an application developed based on embedded Linux environment. It needs to transplant the customized embedded Linux system to the embedded core board with ARMS3C2440 as the core processor. After transplant embedded Linux system to a single user application and the kernel has the communication mechanism [8,9], also have the Web server application running environment, Boa server used in this design, its low occupancy resources, high efficiency, resource constraints into consideration, in the design of embedded Web server dynamic interaction CGI page using the common gateway interface technology, provides a Web server call other executable program interface protocol standard. After the embedded Web server transplantation, the server program development is divided into Web front end and server program design. The main goal of Web front-end program design is to realize user system login and Web user interface at browser end, including user login account verification and HTML dynamic Web page monitoring interface program design. The page style and layout of the monitoring interface USES CSS[10]. When the user clicks the button, an input event will be triggered and sent to the embedded Web server. The Web server will read 
and write according to the input label, and the corresponding serial control data packet will be read through the serial port function for the lower computer. 's main goal is to create a server program design related threads continuously parsing upload serial data packets and refresh to front-end Web page, stored in the local database of the target directory, receiving front-end Web page control events and generate the corresponding command packet downlink transmission, and reads the data oriented to the browser text display control. The embedded Web server program design flow chart is shown in figure 4 .

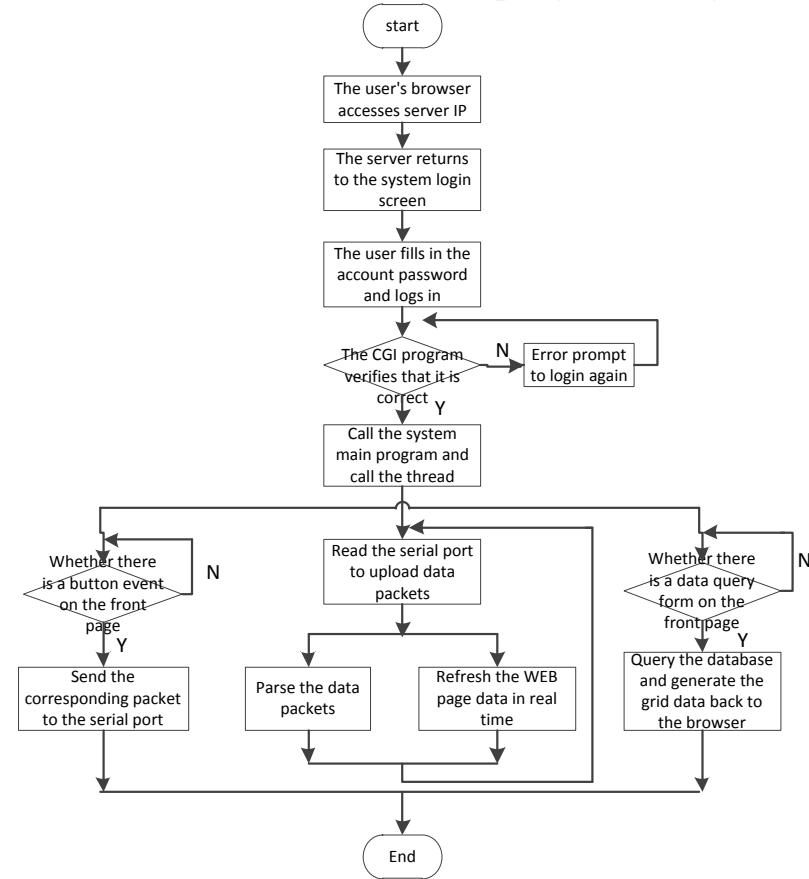

Figure 4. Embedded Web server programming

\section{Remote monitoring system test}

After completing the overall design of the embedded remote monitoring system, the overall performance of the system will be tested, and the remote monitoring simulation remote control terminal will be built. The PLC equipment will be the monitored terminal to test and analyze the performance of the monitoring system. The S3C2440 core board has been ported to embed Linux and Web server programs, and the boa server program is set to self-starter and set local IP address. Monitoring terminal, use Web remote control for PLC equipment, everything is normal after open the remote PC devices, open the browser login embedded server, enter the account password into a Web page interface control system, smoothly into the control interface, can be observed in remote Web page in the form of a system, real-time data were collected system remote login page as shown in figure 5 .

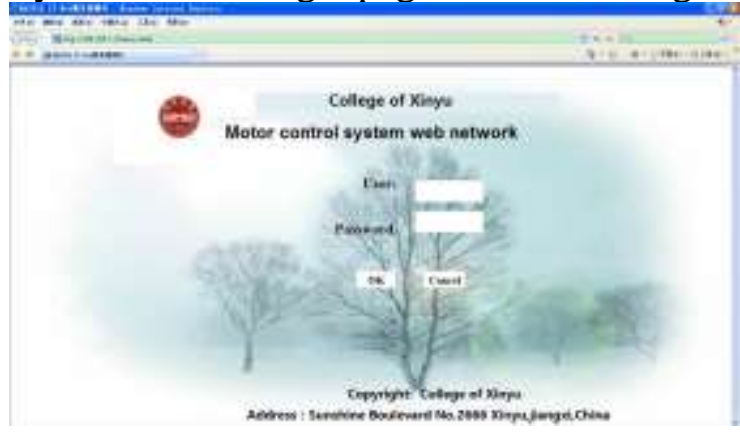

Figure 5. System remote login page

The stability of the remote monitoring system is an important index of the system. When the system is subjected to an uncertain input from the outside or when the system parameters are not allocated properly, it will cause the system to oscillate or possibly deviate from the normal work, and the balance position will be lost. Loss of control command or data in a remote control system. Stability test of this monitoring system is mainly refers to the control terminal control instruction data when monitoring in the network forwarding the packet loss will appear and appear when the ratio of the number of the loss of all forward packets and control instructions in the network transmission is in the form of packets, 
packets during transmission packet loss leads to command appears deviation will affect stability and accuracy of the monitoring system, so the need for stability test system. After repeated tests, each control end can send control command, and the loss rate of control data received by the test end is less than $0.1 \%$. The system loss rate meets the requirements and the system is stable.

\section{Summary}

This paper designs a remote monitoring system of PLC equipment based on embedded Web server, and introduces the system design idea and implementation process from the aspects of system overall design, hardware design and embedded Web server software design. Integrated the hardware resources of ARM embedded platform, designed the embedded Web server, solved the network access problem of the equipment, realized the function of remote control, and met the need of personalized customization of remote control. In the actual test, the system meets the design objective, the system network transmission delay is low, the real-time is good, and realizes the automation, networking acquisition and control of PLC equipment.

\section{Acknowledgements}

provincial department of education science and technology project of Jiangxi 2017 Project No. GJJ171076

\section{References}

[1] Liangjun Sun, Xiuao Hu. HTML + CSS + Java Script web design and layout practical tutorial [M]. Beijing: Tsinghua university press, 2012.(In Chinese)

[2] Ning Lei. Zero-based HTML + CSS [M]. Beijing: mechanical industry press, 2009.(In Chinese)

[3] Xingwei Yang. Embedded WEB server based on ARM-Linux [J]. Programmable controller and factory automation, 2010 (4) : 83-85.(In Chinese)

[4] Sahani M,Nayak A,Agrawal R,et al.A GSM,WSN and embeddedWeb Server Architecture for Internet Based Kitchen Monitoring System[J].2015 International Conference on Circuit,Power and Computing.

[5] Hongan Wei, Yiwen Xu, Zhitao Jin. Research and development of embedded system Web server application [J]. Microcomputers and applications, 2011,30 (11) : 63-65, 69. (In Chinese)

[6] Hailong Wang, Xiaohui Xu, Meng Wang. Implementation of remote control system based on embedded Web server [J].Electronic design engineering, 2010,18 (5) :101-103. (In Chinese)

[7] Si Cheng, Jiaxing Cheng. Study on tunnel technology in VPN [J]. Computer technology and development 2010, (02): 156-159. (In Chinese)

[8] Teubler T, Hail M A, Hellbruck H.Transparent Integration of Non-IP WSN into IP Based Networks [C]//2012 IEEE 8th International Conference on Distributed Computing in Sensor Systems, Hangzhou, 2012: 353-358.

[9] Li Wang, Wei Zhou. Embedded Web server design based on ARM [J]. Computer engineering and applications, 2012,14:90-93,213. (In Chinese)

[10]Longma studio. HTML+CSS+Java Script web page making from novice to expert [M]. Beijing: people's posts and telecommunications press, 2014: 39-122. (In Chinese) 